\title{
House dust mite barrier bedding for childhood asthma: randomised placebo controlled trial in primary care [ISRCTN63308372] Aziz Sheikh*1, Brian Hurwitz ${ }^{1}$, Bonnie Sibbald ${ }^{2}$, Greta Barnes ${ }^{3}$,
Maggie Howe $^{3}$ and Stephen Durham
}

Address: ${ }^{1}$ Department of Primary Health Care \& General Practice, Imperial College of Science, Technology \& Medicine, London, UK, 2National Primary Care Research \& Development Centre, University of Manchester, Manchester, UK, ${ }^{3}$ National Respiratory Training Centre, Warwick, UK and ${ }^{4}$ Upper Respiratory Medicine, Imperial College of Science, Technology \& Medicine, London, UK

E-mail: Aziz Sheikh* - aziz.sheikh@ic.ac.uk; Brian Hurwitz - brian.hurwitz@kcl.ac.uk; Bonnie Sibbald - bsibbald@fs1.cpcr.man.ac.uk; Greta Barnes - gretabarnes@care4free.net; Maggie Howe - m.howe@nrtc.org.uk; Stephen Durham - H.Turner@rbh.nthames.nhs.uk

* Corresponding author

Published: 18 June 2002

Received: 9 May 2002

BMC Family Practice 2002, 3:12

Accepted: 18 June 2002

This article is available from: http://www.biomedcentral.com/I47I-2296/3/12

(C) 2002 Sheikh et al; licensee BioMed Central Ltd. Verbatim copying and redistribution of this article are permitted in any medium for any purpose, provided this notice is preserved along with the article's original URL.

\begin{abstract}
Background: The house dust mite is the most important environmental allergen implicated in the aetiology of childhood asthma in the UK. Dust mite barrier bedding is relatively inexpensive, convenient to use, and of proven effectiveness in reducing mattress house dust mite load, but no studies have evaluated its clinical effectiveness in the control of childhood asthma when dispensed in primary care. We therefore aimed to evaluate the effectiveness of house dust mite barrier bedding in children with asthma treated in primary care.
\end{abstract}

Methods: Pragmatic, randomised, double-blind, placebo controlled trial conducted in eight family practices in England. Forty-seven children aged 5 to 14 years with confirmed house dust mite sensitive asthma were randomised to receive six months treatment with either house dust mite barrier or placebo bedding. Peak expiratory flow was the main outcome measure of interest; secondary outcome measures included asthma symptom scores and asthma medication usage.

Results: No difference was noted in mean monthly peak expiratory flow, asthma symptom score, medication usage or asthma consultations, between children who received active bedding and those who received placebo bedding.

Conclusions: Treating house dust mite sensitive asthmatic children in primary care with house dust mite barrier bedding for six months failed to improve peak expiratory flow. Results strongly suggest that the intervention made no impact upon other clinical features of asthma.

\section{Background}

Asthma is now the commonest chronic disease of childhood in the UK and evidence strongly suggests recent increases in prevalence and severity of the condition. [1-3] Aetiologically, changes to the indoor environment have been implicated in both these increases; proliferation in
British homes of soft furnishings, wall-to-wall carpets, central heating, and double glazing, together with the tendency for children to spend more time indoors, have all combined to increase exposure to airborne indoor allergens. [4] This pathophysiological element in the aetiology of asthma is reflected in the current British Thoracic Soci- 
ety Guidelines on Asthma Management which advise allergen avoidance should be considered in the treatment of all patients with asthma.[5]

The allergen most commonly implicated in the UK is house dust mite, mattresses, bedding, and carpets forming its most important domestic reservoirs. A number of approaches to decreasing house dust mite exposure have been tried, of which house dust mite impermeable bedding is the most promising.[6] New semi-permeable (microporous) barrier bedding interventions are convenient and offer several potential advantages including easy compliance, a preventive approach to individual disease control and relatively low cost (Allerayde single bed set $€ 153$ + VAT).

Though routine use of bedding interventions in dust mite sensitive children with asthma is both logical and safe, uncertainty remains about its clinical effectiveness.[7] The majority of children with asthma are managed almost exclusively in general practice, where the potential role of barrier bedding is unknown. We sought to evaluate the effectiveness of semipermeable house dust mite barrier bedding in children sensitised to the dust mite in a placebo controlled trial conducted from primary care.

\section{Methods}

Study sample, inclusion and exclusion criteria

The study was conducted between 1998 and 1999 in eight group practices recruited from the database of the National Respiratory Training Centre. Practices satisfying the study inclusion criteria were those that operated a dedicated nurse-led asthma clinic, and employed nurses who had received formal training in asthma and allergy on courses run by the National Asthma \& Respiratory Training Centre. Ethical approval was obtained from the North London Multi-centre and relevant Local Research Ethics Committees.

Parents of children aged 5-14 years with a recorded diagnosis of asthma and who had been prescribed one or more asthma treatments in the preceding six months, were contacted by telephone. Children with a clinical history suggestive of house dust mite allergy, as judged by a positive response to the question 'has your child's asthma ever got worse when the bed is made or when the vacuuming or dusting is done?' were offered skin prick tests by practice nurses. Prick tests were performed on the volar aspect of the forearm using standardised ALK Soluprik extracts of Dermatophagoides pteronyssinus (Der p1) and using positive (histamine dihydrochloride) and negative (allergen diluent) controls and were measured at 15 minutes. A positive response to Der p1 was defined as a weal of at least five millimetres greater than that induced by the negative control. Only children with both subjective and objective evidence of allergy to the house dust mite were invited to participate. Exclusion criteria were dermographism (because of the difficulty in interpreting skin prick test results), children who did not use a duvet, those already using allergy control bedding, and cat or dog ownership. Pet owners were excluded because co-sensitivity in dust mite sensitive individuals is common and the additional allergen load from the pet could swamp any effect of the barrier bedding. Written consent from parents and verbal consent from children aged seven and over was obtained in all cases.

\section{Randomisation and intervention}

To eliminate possible allocation bias centralised randomisation of individual patients was performed using numbers generated from a random numbers table. Bedding sets comprising mattress, duvet and pillow covers were posted from the randomisation centre directly to the homes of recruited children. Bedding consisted of either Allerayde Perfect house dust mite impermeable covers or identical looking placebo covers produced by the same company, and was supplied to patients in the study free of charge, to be used for six months. Both subjects and nurses were blinded to which trial arm children were allocated to, as were those responsible for data analysis; the trial code was broken after data analysis had been completed. To ensure that only the effect of the bedding was being evaluated all patients were given identical advice and written instructions regarding ways to minimise house dust mite exposure at home.

Parents were asked to keep a daily symptom diary for their child detailing individual asthma (cough, wheeze, shortness of breath and chest tightness) and rhinitis (sneeze, runny nose, blocked nose) symptoms on a four-point Likert scale (0-3). Each symptom score was tallied and summed together to produce a 28-day composite score for asthma, and for rhinitis. Such symptom scores could vary between 0 (no symptoms) and 336 for asthma, between 0 (no symptoms) and 252 for rhinitis. Details of daily usage of asthma medication and episodes of night-time waking were also recorded.

Each practice nurse reviewed children at monthly intervals for six months, measured peak expiratory flow (recording the best reading from three attempts), assessed asthma control, and asked parents whether the bedding covers issued were still being used. At the two-month post-intervention assessment, and thereafter, patients whose asthma control was considered good by the nurses were advised to reduce inhaled steroid use by $50 \%$, in the context of clear instructions regarding steps to be taken should asthma symptoms deteriorate. In the event of acute exacerbations of asthma nurses recommended a standardised protocol regarding indications for use of oral 
steroids, which were to be used in consultation with the general practitioner.

The initial assessment, involving skin prick testing, was performed in the patient's home. Thereafter, children were seen at monthly intervals in the practice asthma clinic until the final assessment, which was performed at the child's home. On completing the study, nurses checked paper and electronic medical records to extract data on all non-study related practice consultations, and accident $\&$ emergency department consultations for asthma, hospital admissions for asthma, and details of the number of courses of oral steroids prescribed during the study period.

\section{Statistical methods}

To detect an average within person change of $10 \%$ or more in peak expiratory flow (PEF) from baseline reading at the start of the study with $80 \%$ power at the $5 \%$ significance level (assuming a within person standard deviation of $35 \mathrm{l} / \mathrm{min}$ ), we estimated that 21 patients were needed in each arm of the study. Normally distributed data were analysed using student $\mathrm{t}$-test and mean values and standard deviations calculated; the Mann Whitney test was used for non-parametric data and medians and inter-quartile ranges calculated. Categorical data were analysed using Chi-square test or Fisher's exact test in the event of small numbers. SPSS Windows Version 7.5 was used for all statistical analyses.

\section{Results}

\section{Baseline data}

The combined list size of study practices was 55,687, of whom 6095 patients were aged 5-14 years of whom 1157 (19\%) had a recorded diagnosis of asthma. Four hundred and seventy two children were approached to join the study 47 of whom satisfied the inclusion criteria. Reasons for excluding patients are summarised in Table 1.

Table I: Reasons for excluding children

\begin{tabular}{ll}
\hline Reason for exclusion & n (\%) \\
\hline & \\
Lack of parental interest & $123(29)$ \\
Pet ownership & $106(25)$ \\
No asthma medication in previous 6 months & $89(21)$ \\
Skin prick test <5 mm to house dust mite & $64(15)$ \\
Already using allergy control bedding & $17(4)$ \\
Child not using a duvet & $9(2)$ \\
Child not sleeping in own bed & $4(1)$ \\
Unrecorded & $13(3)$ \\
Total & $\mathbf{4 2 5}$ \\
& \\
\hline
\end{tabular}

Forty-three children (92\%) completed the study (Figure 1), all of whom reported use of bed covers supplied for the duration of the trial. Three of the children lost to followup failed to attend for scheduled reviews; parents of the fourth child stated they no longer wished to participate. Mean age of children was 11.0 years (sd 2.26) with a preponderance of boys $(62 \%)$. The treatment arm contained $26(55 \%)$ children and the placebo group 21 (45\%). Details of age, sex, baseline asthma symptom scores, medication usage, and peak expiratory flow measures for each group are given in Table 2. There was no major difference between groups in baseline skin prick test weal diameter to house dust mite, or in the proportion of households with a smoker, bedroom central heating, double-glazing or wall-to-wall carpets.

\section{Peak expiratory flow, symptom scores, and health care uti- lisation}

Comparisons of changes in peak expiratory flow and other clinical data are summarised in Table 3 . The results show that whilst mean peak expiratory flow increased in both groups when compared to baseline, there was no significant difference in the increases achieved when comparing active and placebo treated groups. Although mean asthma, rhinitis and night-time waking scores decreased from baseline in both groups, differences were not significant when comparing placebo and active treatment arms. Of interest is that these tendencies towards improvement in symptomatic and clinical outcomes, when compared to baseline, were seen in the context of reductions in mean inhaled corticosteroid usage when compared with baseline usage. Only four children (two in each arm) required courses of oral steroid throughout the study and there were no asthma-related hospital admissions during this time period.

\section{Discussion}

In a carefully selected population of HDM sensitive children 6 months community based treatment with allergy control bedding achieved no significant improvement in peak expiratory flow in those treated with impermeable bedding covers compared with those treated with placebo covers. The trial results also suggest that HDM impermeable covers made no impact on other clinical features of asthma. The trial was sufficiently powered to detect a clinically significant improvement for our main outcome measure of interest, peak expiratory flow, but this is not true of the other outcome measures studied. One possible reason for the lack of effect is that families did not comply with the intervention. Although we did not measure compliance directly, self-reported compliance was very good, and nurses did not report any lack of bedding usage in home assessments undertaken at the end of the study when each child's bed was inspected to see if the covers were still in place. Another possible explanation for the 
Table 2: Baseline comparison between treatment and placebo groups

\begin{tabular}{lll}
\hline Variable & HDM impermeable bedding, $\mathbf{n}=\mathbf{2 6}$ Placebo bedding, $\mathbf{n}=\mathbf{2} \mathbf{I}$ \\
\hline & & \\
Age, mean (sd) & $10.58(2.34)$ & $11.57(2.08)$ \\
Males (\%) & $16(62)$ & $13(62)$ \\
Peak expiratory flow in litres/min, mean (sd) & $288.80(100.80)$ & $328.57(73.02)$ \\
Number on inhaled steroids (\%) & $5(19.2)$ & $5(23.8)$ \\
28-day asthma symptom score (0-336), median (inter-qaurtile range) & $12.0(6.5$ to 52.5$)$ & $37.0(9.0$ to 69.0$)$ \\
28-day rhinitis symptom score (0-252), median (inter-quartile range) & $50.5(19.8$ to 82.5$)$ & $51.0(23.5$ to 84.5$)$ \\
Episodes of night-time waking over 28 days, median (inter-quartile range) & $0(0$ to 0.75$)$ & $0(0$ to 3.0$)$ \\
28-day dose inhaled steroid mcg/month, median (inter-quartile range) & $5600(1450$ to II,100) & $5400(58$ to II,200) \\
28-day dose bronchodilator mcg/month, median (inter-quartile range) & $800(125$ to 1700$)$ & $400(100$ to 42$)$
\end{tabular}

Table 3: Comparison of changes from beginning to end of study in clinical outcomes between treatment and placebo groups

\begin{tabular}{|c|c|c|c|c|}
\hline Medical outcome variable & $\begin{array}{l}\text { HDM Impermea- } \\
\text { ble bedding, } n=23\end{array}$ & $\begin{array}{l}\text { Placebo bedding, } \\
n=20\end{array}$ & $\begin{array}{l}95 \% \mathrm{Cl} \text { of differ- } \\
\text { ence }\end{array}$ & Significance $p$ \\
\hline Mean change in 28-day PEF litres/min (sd) & $16.38(25.62)$ & $13.68(43.14)$ & -25.15 to 19.75 & 0.81 \\
\hline Mean change in 28 -day asthma symptom scores (sd) & $-3.40(29.50)$ & $-18.10(27.80)$ & -33.16 to 3.86 & 0.12 \\
\hline Mean change in 28-day rhinitis symptom scores (sd) & $-31.14(35.79)$ & $-22.67(30.70)$ & -13.15 to 30.09 & 0.43 \\
\hline $\begin{array}{l}\text { Mean change in episodes of monthly night-time waking over } \\
\text { 28-day period (sd) }\end{array}$ & $-0.64(3.00)$ & $-0.94(2.30)$ & -2.05 to 1.93 & 0,43 \\
\hline Mean change in 28 -day dose of inhaled steroids mcg (sd) & $-|8| 5.9 \mid(386 \mid .45)$ & $-1039.00(1881.15)$ & $\begin{array}{l}-1128.58 \text { to } \\
2682.59\end{array}$ & 0.41 \\
\hline Median non-study GP consultations (range) & $I(0-5)$ & $0(0-5)$ & & 0.26 \\
\hline Total number of hospital admissions for asthma & 0 & 0 & & I \\
\hline Total number of courses of oral steroids for asthma & 2 & 2 & & I \\
\hline
\end{tabular}

lack of effect is that the pragmatic intervention employed failed to reduce house dust mite load sufficiently. Due to an ambiguity in the sampling protocol, we were unable, in this study, to obtain valid information on post-intervention house dust mite loads, but data from other studies show bedding covers of the sort we supplied to be highly effective in reducing mattress dust mite load.[8] A further possible explanation could be that the patients recruited on average suffered relatively mild disease (reflected by their low baseline asthma symptom scores); they were therefore sufficiently well controlled on existing treatment for little scope to remain for clinical improvement (Table 2).

The majority of asthmatic children managed in primary care have mild to moderate disease; in an attempt to maximise the chances of detecting clinical improvement we only recruited children who had active asthma (as evidenced by recent prescription for asthma medication) in whom attempts were made to reduce inhaled steroid use in a standardised manner. We used PEF as our primary outcome measure of interest since this is the objective measure most commonly used by general practitioners and paediatricians; spirometry would not have been feasible in such young children. Because of concerns regarding the validity of self-recorded PEF in children, however, we opted for nurse-supervised clinic based recording rather than early morning PEF, another possible explanation for our failure to detect a difference. Finally, there is the possibility that the intervention reduced dust mite load but this failed to produce measurable clinical impact on patients.[9]

Allergen avoidance measures are likely to be of most benefit in patients truly sensitive to the allergen in question. We therefore only included children with unequivocal allergy to the house dust mite as determined by both a subjective clinical history and objective positive weal and flare response on skin prick testing. Our findings suggest firstly that allergy to this organism, defined by a positive 


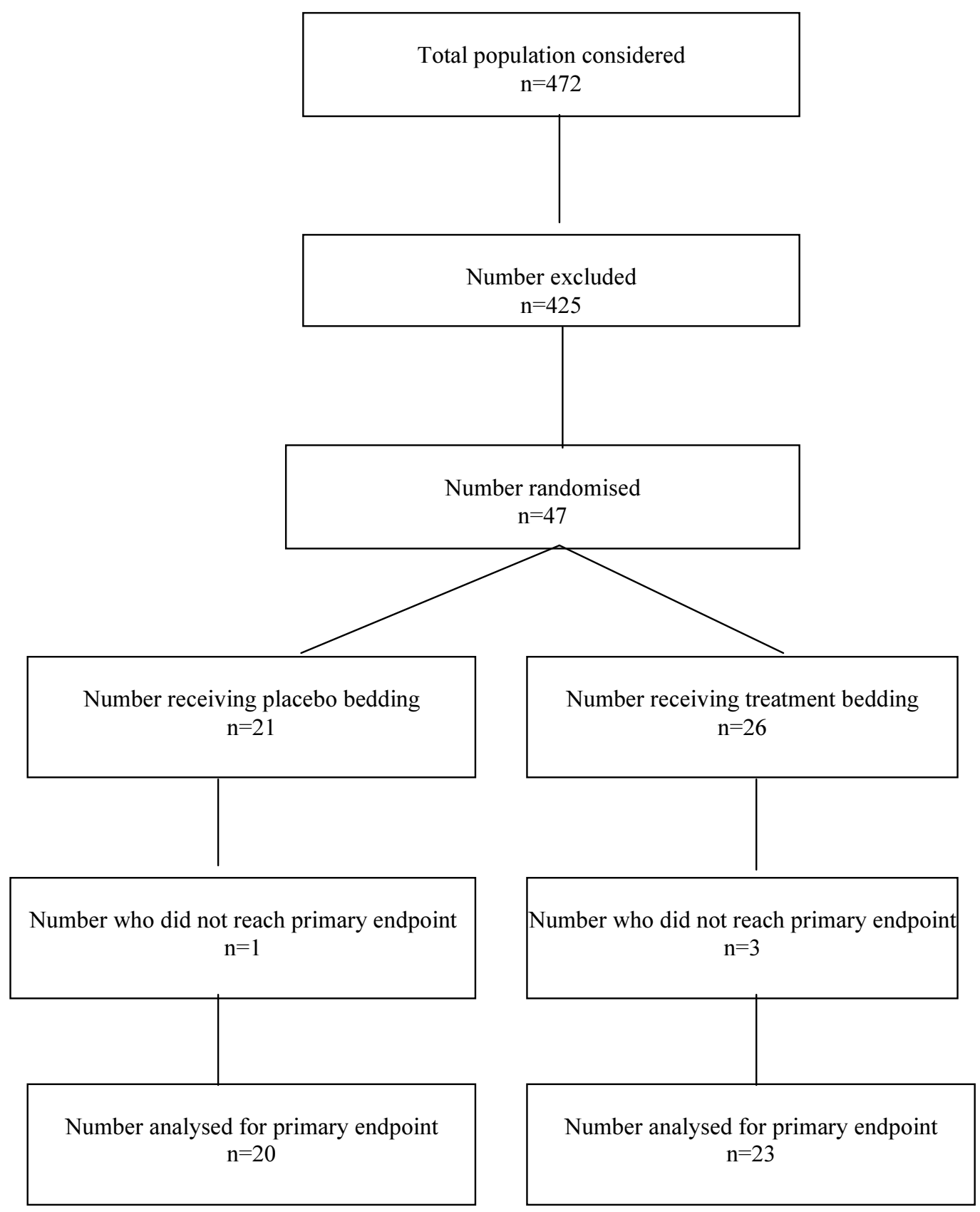

Figure 1: CONSORT flow diagram

Figure I 
history and skin prick test of five millimetres or more, may not be as prevalent in GP registered populations as previously suspected. Clinical history alone appears to have low predictive value for allergy to dust mite in asthmatic children, [10] and house dust mite barrier bedding has at most only a minor impact on health outcomes for children with mild asthma in non pet owning families managed in general practice.

The rationale for offering patients advice in general practice about house dust mite avoidance for asthma (or for other house dust mite associated conditions such as perennial rhinitis) requires recourse to objective tests such as skin prick testing. Very few general practitioners currently perform skin prick testing, lacking both the training and financial support to do so.[11] The alternative of radio-allergo absorbent testing (RAST) is more expensive.

Our study adds support to the conclusions of a recent meta-analysis of house dust mite avoidance measures in asthma patients, which concluded that existing interventions designed to decrease domestic dust mite exposure are of little, if any, clinical benefit.[12] Our results, in conjunction with those of the meta-analysis, lead us to question whether routine use of house dust mite barrier bedding as treatment for mildly affected asthmatic children can be justified in the clinical setting of general practice. Further explanatory and pragmatic intervention studies are required, to estimate the value, if any, of mite reduction strategies in mite sensitive asthmatic children with more severe disease.

\section{Conclusions}

In a carefully selected population of HDM sensitive children, 6 months community based treatment with allergy control bedding achieved no significant improvement in peak expiratory flow in those treated with impermeable bedding covers compared with those treated with placebo covers. Until the results of other trials evaluating the role of house dust mite barrier bedding become available, we recommend that topical corticosteroids remain the treatment of choice in those children with persistent asthma symptoms.

\section{Competing interests}

AS has received a small travel grant from Allerayde, a manufacturer of house dust mite barrier bedding.

\section{Authors' contributions}

AS was responsible for the day-to-day management of the project, this involving contributing to the design of the study protocol, obtaining ethics approval, data collection and analysis, and co-ordinating the writing up of the paper. BH contributed to design of study protocols, interpretation of results and writing up of the manuscript. BS conceived the idea for this project, contributed to design of study protocols, interpretation of results and writing up of the manuscript. GB secured funding for the project, contributed to study design and the interpretation of results. MH assisted in co-ordinating the study; she was actively involved in supervising practice recruitment, nurse training and designing study protocols. SD conceived the idea for this project, contributed to the design of study protocols, interpretation of results, and writing up of the paper. All authors read and approved the final manuscript.

\section{Acknowledgements}

We thank the nurses whose dedication made this study possible: Diana Beken, Liz Bryant, Joan Flemming, Frances Fry, Wendy Johnson, Janetta Kennedy, Judith McAllister and Catherine Tutt. Thanks also to Dr Meinir Jones and Dr Paul Cullinan of the National Heart \& Lung Institute for their advice, ALK Abello for supplying the skin prick test kits and solutions and Allerayde for supplying bedding at a subsidised rate. This study was supported by a project grant from the National Respiratory Training Centre, Warwick. AS initiated this study whilst funded by the London Academic Training Scheme; he currently holds a NHS R\&D National Primary Care Training Award.

\section{References}

I. Strachan DP, Butland BK, Anderson HR: Incidence and prognosis of asthma and wheezing illness from early childhood to age 33 in a national British cohort. BMJ 1996, 3 I 2: I I95-II99

2. Lewis S, Butland B, Strachan D, et al: Study of the aetiology of wheezing illness at age 16 in two national British cohorts. Thorax 1996, 5 I:670-676

3. Burr ML, Butland BK, King S, et al: Changes in asthma prevalence: two surveys 15 years apart. Arch Dis Child 1989, 64:1452-1456

4. Woodcock A, Custovic A: Avoiding exposure to indoor allergens. In: $A B C$ of allergies. (Edited by: SR Durham) London: BMJ 1998, 3235

5. British Thoracic Society, National Asthma Campaign, Royal College of Physicians of London. The British guidelines on asthma management: 1995 review and position statement. Thorax 1997, 52:S2-S8

6. Custovic A, Simpson A, Chapman MD, et al: Allergen avoidance in the treatment of asthma and atopic disorders. Thorax 1998 , 53:63-72

7. Strachan DP: House dust mite allergen avoidance in asthma. Benefits unproved but not yet excluded. $\mathrm{Br}$ Med J 1998, 3 17:1096-1097

8. Ehnert B, Lau-Schadendorf S, Weber A, et al: Reducing domestic exposure to dust mite allergen reduces bronchial hyperreactivity in sensitive children with asthma. J Allergy Clin Immunol 1992, 90:35-38

9. Carswell F, Birmingham K, Oliver J, et al: The respiratory effects of reduction of mite allergen in the bedrooms of asthmatic children: a double-blind controlled trial. Clin Exp Allergy 1996, 26:386-396

10. Sibbald B, Barnes G, Durham SR: Skin prick testing in general practice: a pilot study. J Adv Nurs 1997, 26:537-542

I I. Sheikh A, Levy M L: Costs are a barrier to GPs performing skin prick testing. $\mathrm{Br}$ J Gen Pract. 1999, 49:67

12. Gøtzsche PC, Hammarquist PP, Burr M: House dust mite control measures in the management of asthma: meta-analysis. $B M J$ 1998, 3 17:1105-1110

\section{Pre-publication history}

The pre-publication history for this paper can be accessed here:

http://www.biomedcentral.com/1471-2296/3/12/prepub 